\title{
Dynamic Reliability Analysis of Mechanical Components Based on Equivalent Strength Degradation Paths
}

\author{
Peng Gao ${ }^{1}$ - Shaoze Yan ${ }^{1, *}$ - Liyang $\mathrm{Xie}^{2}$ - Jianing $\mathrm{Wu}^{1}$ \\ 1 Tsinghua University, Department of Mechanical Engineering, State Key Laboratory of Tribology, China \\ ${ }^{2}$ Northeastern University, School of Mechanical Engineering, China
}

\begin{abstract}
Owing to the randomness of load applied to mechanical components, it is difficult to accurately determine the strength degradation path. Therefore, the distribution of strength at each load application is always used to deal with the uncertainty of strength in its degradation process, which may cause errors in reliability calculations due to neglecting the correlation with the remaining strength at each load application in a strength degradation path. To deal with this problem, dynamic reliability models of mechanical components with the failure mode of fatigue are developed in this paper, based on equivalent strength degradation paths, whose uncertainty is determined by both the distribution of material parameters and the distribution of load. The proposed models can be used to quantitatively analyse the influences of the variation in statistical parameters of material parameters on the reliability and failure rate of components. Explosive bolts, which are important to the successful launch of satellites, have been chosen as representative examples to validate the effectiveness and accuracy of the proposed models. The results show that using strength distribution at each load application may lead to large errors in calculating reliability. Moreover, different material parameters have different influences on dynamic characteristics of reliability and on the failure rate of mechanical components.
\end{abstract}

Keywords: dynamic reliability, correlation, remaining strength, mechanical components, strength degradation path

\section{INTRODUCTION}

In the design stage of mechanical components, the uncertainty in both the environmental load and the material parameters needs to be taken into consideration and a safety margin is required to guarantee the intrinsic reliability of mechanical components. The safety factor is comprehensively used in the practical design of mechanical components, which is industry specific and determined by the experience of engineers. However, the empirical safety factor cannot quantify the uncertainty and risk in mechanical design. Therefore, reliability analysis of mechanical products has gained more and more popularity [1] to [3].

Reliability is defined as the probability that a product performs its intended functions without failure during a specified time period. For mechanical components, the load-strength interference (LSI) model is the most important analytical method in reliability assessment. The conventional LSI model is essentially a static reliability model. However, gradual failure of mechanical components commonly exists in practical engineering due to the strength degradation caused by corrosion, wear, erosion creep, etc. As pointed out by Martin, constructing reliability models considering strength degradation is an important issue for reliability estimation and further research on generalized methods for the dynamic reliability analysis of mechanical components is imperative [4].
To overcome the shortcomings of conventional LSI models, reliability models based on stochastic process theory are investigated in which load and strength are modelled as two stochastic processes. Lewis [5] analysed the time-dependent behaviour of a 1-out-of-2: G redundant system by combining the LSI model with a Markov model. Geidl and Saunders[6] introduced time-dependent elements into the reliability equation to estimate the reliability. Somasundaram and Dhas[7] put forward a generalized formula to estimate the reliability of a dynamic parallel system, in which components equally shared the load. Noortwijk and Weide [8] developed a reliability model, in which load and strength are described as two stochastic processes. Labeau et al. proposed the framework of a dynamic reliability platform and identified its main constituents [9]. Zhang et al. analysed the main methods for dynamic reliability estimation of nuclear power plants, which include discrete dynamic event trees and Monte Carlo simulation [10]. Slak analysed production planning and scheduling, cutting tools and material flow process, and manufacturing capacities [11]. Barkallah et al. proposed a method for process planning to determine the tolerance for manufacturing with statistical tools [12].

As a matter of fact, the reliability models based on stochastic process theory, such as the timedependent model, Markov model, etc, are the most important tools for dynamic reliability analysis. The Markov models are mainly used for dynamic reliability analysis of electronic elements and multi- 
state systems. In the Markov models, the elements and the systems are characterised by different states at different time instants and the dynamic reliability is calculated based on the state transition matrix between two different time instants. However, it is very difficult to define and diagnose the states of mechanical components. In addition, the strength of mechanical components degrades under the application of environmental load. The state-based reliability models cannot be used for further investigation of how stress and material parameters influence the reliability of mechanical components.

In addition, much progress has been made in dynamic reliability analysis based on time-dependent models. The essence of the time-dependent models based on stochastic process theory is to calculate reliability by assuming the stress process and the strength degradation process to be two continuous processes, which are mathematically expressed as various stochastic processes. However, for mechanical components in the failure mode of fatigue, some problems exist in directly extending these reliability models to the dynamic reliability analysis of mechanical components and these are listed as follows:

(1) For mechanical components with the failure mode of fatigue, the strength degradation process is discontinuous. In this case, it is meaningless to analyse the reliability at a given time instant. A more detailed explanation will be given in Section 1. The dynamic reliability analysis should be performed for a given time interval or at a specified application of load. Therefore, it is more straightforward and reasonable to establish dynamic reliability models with respect to load application times, which can provide the basis for developing dynamic reliability models with respect to time. However, dynamic reliability models with respect to load application times considering strength degradation have not been widely used.

(2) For the convenience of developing a dynamic reliability model, various stochastic processes are assumed to model the strength degradation process in the time-dependent models without further explanation of the physical significance of the parameters involved in the strength processes. However, these proposed dynamic reliability models cannot be used to analyse how the reliability is influenced by the variation in the statistical parameters of material parameters.

(3) Due to the randomness of load magnitude at each application, it is difficult to determine the strength degradation path. Therefore, the distribution of strength at each time instant or each load application is always used for reliability calculations in the time- dependent models, which can be derived based on the properties of the assumed strength degradation process. However, it may cause large errors to calculate reliability in this way due to neglecting the correlation between the remaining strength at each load application, something that is not pointed out in current literature.

To deal with these problems, this paper proposes dynamic reliability models that take into account the degradation mechanism of mechanical components and that can be used to quantitatively analyse the influence of the variation in the statistical characteristics of material parameters on the dynamic behaviour of reliability and failure rate of mechanical components. In the proposed models, the stress, strength, and load application times are modelled as random variables. Moreover, the proposed reliability models are established based on an analysis of the strength degradation path rather than on the distribution of strength at each load application.

\section{RELIABILITY MODELS WITH RESPECT TO APPLICATION TIMES OF RANDOM LOAD}

Unlike the failure mode of corrosion, when only the failure mode of fatigue is taken into consideration, the load process is a discrete process. The assumption that the statistical characteristics of load are continuous functions of time indicates that there are infinite times of load application in an infinitesimal time interval $\Delta t$, which is obviously unreasonable in reality. Therefore, the load process should be characterised by load application times and magnitude of load. In addition, under the failure mode of fatigue, strength does not degrade in the absence of load application and the change in strength is discontinuous as shown in Fig. 1.

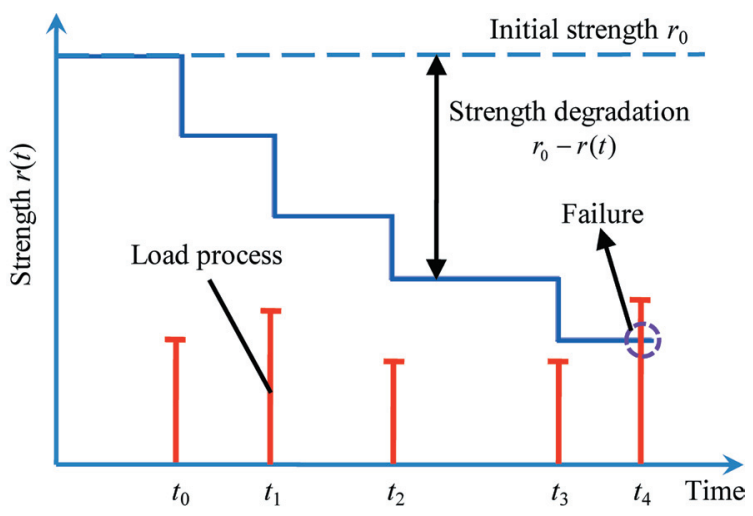

Fig. 1. Schematic process of strength degradation

From Fig.1, it can be seen that at any time instant between two adjacent load applications, the reliability 
of the components is equal to one, which is different from the reliability in a given time interval. Hence, the reliability analysis at a given time instant is meaningless for the reliability analysis of mechanical components with the failure mode of fatigue. It is more straightforward and reasonable to model strength as a function of load application times rather than directly as the function of time. However, dynamic reliability models with respect to load application times considering strength degradation are not widely used. In this section, the dynamic reliability models of mechanical components with respect to load application times are developed, which provide the basis for dynamic reliability analysis with respect to time. Besides, the influences of the statistical characteristics of load and material parameters on reliability and failure rate are analysed.

\subsection{Reliability Models with Respect to Load Application Times}

Due to the randomness of magnitude of load at each application, it is difficult to describe the degradation path of strength. Therefore, the method of calculating dynamic reliability based on the strength distribution at each load application, which is determined according to the assumed stochastic process of strength degradation, is adopted with all the features. However, this may lead to wrong estimates in calculating the reliability using the strength distribution at each load application, because some impossible strength degradation paths are included in the reliability calculation. For illustrative convenience, we list some possible strength degradation paths in Fig. 2. The uncertainty of the strength degradation path comes from the randomness of load magnitude at each load application. In Fig. 2, a circle represents a possible changing point of strength at a load application. Therefore, the strength degradation path can be characterised by the changing points. The possible paths in Fig. 2 are summarised in Table 1.

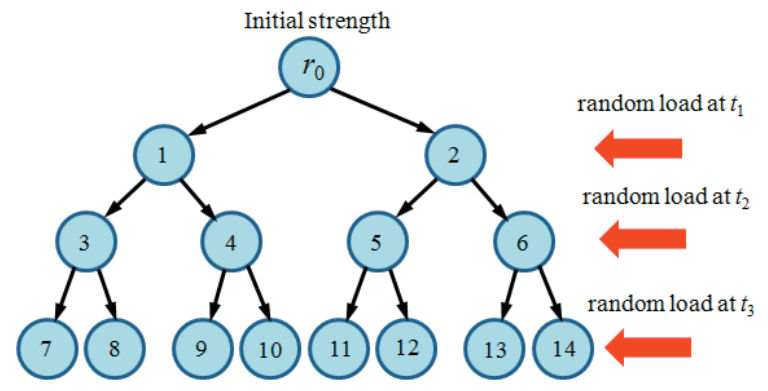

Fig. 2. Strength degradation path
Table 1. Strength degradation path

\begin{tabular}{cccc}
\hline Strength & \multicolumn{3}{c}{ Changing point } \\
\cline { 2 - 4 } degradation path & $t_{1}$ & $t_{2}$ & $t_{3}$ \\
\hline$r_{0}-1-3-7$ & 1 & 3 & 7 \\
\hline$r_{0}-1-3-8$ & 1 & 3 & 8 \\
\hline$r_{0}-1-4-9$ & 1 & 4 & 9 \\
\hline$r_{0}-1-4-10$ & 1 & 4 & 10 \\
\hline$r_{0}-2-5-11$ & 2 & 5 & 11 \\
\hline$r_{0}-2-5-12$ & 2 & 5 & 12 \\
\hline$r_{0}-2-6-13$ & 2 & 6 & 13 \\
\hline$r_{0}-2-6-14$ & 2 & 6 & 14 \\
\hline
\end{tabular}

From Table 1, it can be seen that there are two possible changing points of strength (1-2), four possible changing points of strength (3-6) and eight possible changing points (7-14) at $t_{1}, t_{2}$ and $t_{3}$, respectively. When calculating reliability by using the strength distribution at each load application, paths with all possible combinations of changing points of strength are taken into account, which also include impossible paths, such as path $r_{0}-1-6-10$, path $r_{0}$-2-4-12, etc. Therefore, the method based on the distribution of strength at each load application may result in errors in the assessment of reliability, which will be illustrated later. An alternative method for dynamic reliability analysis is to perform a Monte Carlo simulation, in which the strength degradation is simulated based on the degradation mechanism of mechanical components and the random loads are generated according to their probability distributions. However, the Monte Carlo simulation is considerably time consuming and the time for a Monte Carlo simulation increases rapidly with the increase in load application times, which limits the practical applicability of this simulation. In addition, the Monte Carlo simulation method cannot be used to quantitatively analyse the influences of the variation in statistical parameters of material parameters on the reliability and failure rate of mechanical components.

To deal with these problems, dynamic reliability models with respect to load application times are developed in this section, which can then be used to calculate the reliability of mechanical components using the failure mode of fatigue under the application of random load for arbitrary times.

The strength degradation path for a given sample mechanical component is deterministic. In general, the remaining strength of the mechanical components can be expressed in the following form [13].

$$
r(n)=r_{0}[1-D(n)]^{a},
$$


where $n$ and are the load application times and initial strength, respectively, and $a$ is the material parameter. $D(n)$ is the cumulative damage caused by load, which is determined by both load application times $n$ and the magnitude of load. According to the Miner linear damage accumulation rule [14], the damage caused by a load with a magnitude of once is:

$$
D_{i}\left(n_{i}\right)=\frac{1}{N_{i}},
$$

where $N_{i}$ is the lifetime of a component under a load with a magnitude of $s_{i}$. Analogously, the damage caused by a load with a magnitude of $s_{0}$ once is:

$$
D_{0}(1)=1 / N_{0},
$$

where $N_{0}$ is the lifetime of a component under the load with the magnitude of $s_{0}$. According to the $S$ - $N$ Curve theory of components, the relationship between the load $s_{i}$ applied to a components and the corresponding lifetime $N_{i}$ of the component under the application of $s_{i}$ can be mathematically expressed as follows:

$$
s_{i}^{m} N_{i}=C,
$$

where $m$ and $C$ are material parameters and the dispersion of lifetime is represented by the dispersion of the parameter $C$. Similarly, the relationship between and $N_{0}$ can be written as:

$$
s_{0}{ }^{m} N_{0}=C
$$

From Eq. (4) and Eq. (5), it can be derived that:

$$
D_{i}(1)=\frac{1}{N_{i}}=\frac{s_{i}^{m}}{C}
$$

and

$$
D_{0}(1)=\frac{1}{N_{0}}=\frac{s_{0}{ }^{m}}{C} \text {. }
$$

Therefore, from Eqs. (6) and (7), it can be derived that the damage caused by a load with a magnitude of $s_{i}$ once is equal to the damage caused by a load with a magnitude of $s_{0}$ for $n_{i 0}$ times under the condition that:

$$
n_{i 0}=\left(\frac{s_{1}}{s_{0}}\right)^{m}
$$

According to the total probability theorem, the damage caused by the application of a random load with a probability density function (pdf) of $f_{s}(s)$ once can be approximated by the damage caused by a magnitude of $s_{0}$ load applied $n_{0}$ times, which is given by:

$$
n_{0}=\frac{1}{s_{0}{ }^{m}} \int_{-\infty}^{\infty} s^{m} f_{s}(s) \mathrm{d} s
$$

Therefore, for a deterministic initial strength, the remaining strength in an equivalent strength degradation path can be expressed according to Eq. (1) as follows:

$$
\begin{aligned}
r(n) & =r_{0}[1-D(n)]^{a}=r_{0}\left(1-\frac{n_{0} n}{N_{0}}\right)^{a}= \\
& =r_{0}\left(1-\frac{n \int_{-\infty}^{\infty} s^{m} f_{s}(s) \mathrm{d} s}{C}\right)^{a} .
\end{aligned}
$$

For a given component with deterministic initial strength $r_{0}$ and material parameter $C$, the reliability under the application of random load $n$ times can be derived as:

$$
R(n)=\prod_{i=0}^{n-1}\left[\int_{-\infty}^{r_{0}\left(1-\frac{i \int_{-\infty}^{\infty} s^{m} f_{s}(s) \mathrm{d} s}{C}\right)^{a}} f_{s}(s) \mathrm{d} s\right] .
$$

To consider the randomness of the initial strength and the material parameter $C$, we denote the pdf of $r_{0}$ and $C$ by $f_{r 0}\left(r_{0}\right)$ and $f_{C}(C)$, respectively. According to the Bayes law for continuous variables, the reliability of components with respect to load application times considering strength degradation can be expressed as follows:

$$
R(n)=\int_{-\infty}^{\infty} f_{r_{0}}\left(r_{0}\right) \int_{-\infty}^{\infty} f_{C}(C)\left\{\prod_{i=0}^{n-1}\left[\int_{-\infty}^{r_{0}\left(1-\frac{i \int_{-\infty}^{\infty} s^{n} f_{s}(s) d s}{C}\right)^{a}} f_{s}(s) \mathrm{d} s\right]\right\} d C d r_{0} .
$$

According to the definition of failure rate, the failure rate of components with respect to load application times can be written as:

$$
\begin{aligned}
& h(n)=\frac{F(n+1)-F(n)}{R(n)}= \\
& =\left\{\int_{-\infty}^{\infty} f_{r_{0}}\left(r_{0}\right) \int_{-\infty}^{\infty} f_{C}(C)\left\{\prod_{i=0}^{n-1}\left[\int_{-\infty}^{r_{0}\left(1-\frac{i \int_{-\infty}^{\infty} s^{m} f_{s}(s) \mathrm{d} \mathrm{d} s}{C}\right)^{a}} f_{s}(s) \mathrm{d} s\right]\right\} d C d r_{0}-\right. \\
& \left.\left.-\int_{-\infty}^{\infty} f_{r_{0}}\left(r_{0}\right) \int_{-\infty}^{\infty} f_{C}(C)\left\{\prod_{i=0}^{n}\left[\int_{-\infty}^{r_{0}\left(1-\frac{i-\infty}{C} \int_{-\infty}^{\infty} s^{m} f_{s}(s) d s\right.}\right)^{a} f_{s}(s) \mathrm{d} s\right]\right\} d C d r_{0}\right\} / \\
& \left\{\left\{\int_{-\infty}^{\infty} f_{r_{0}}\left(r_{0}\right) \int_{-\infty}^{\infty} f_{C}(C)\left\{\prod_{i=0}^{n-1}\left[\int_{-\infty}^{r_{0}\left(1-\int_{-\infty}^{\infty} \int^{s^{m} f_{s}(s) \mathrm{d} s}\right)^{a}}{ }^{a}(s) \mathrm{d} s\right]\right\} d C d r_{0}\right\} .\right.
\end{aligned}
$$

In the case where strength degradation does not take place, Eq. (12) degenerates into the following form: 


$$
R(n)=\int_{-\infty}^{\infty} f_{r_{0}}\left(r_{0}\right)\left[\int_{-\infty}^{r_{0}} f_{s}(s) d s\right]^{n} d r_{0}
$$

Specifically, when the value of $n$ is equal to 1, Eq. (14) reduces to the conventional LSI model.

\subsection{Numerical Examples}

In this section, explosive bolts are chosen as representative examples to illustrate the proposed reliability models. Explosive bolts are of considerable importance to the successful launch of satellites as a connection and pyrotechnical separation device. The structure of the explosive bolt is shown in Fig. 3 [15]. In the launch process of satellites, the explosive bolt is used for the connection between the payload adapter and the interface ring, which are connected to the launch vehicle and the satellite, respectively. In the departure process of satellites and launch vehicles, the explosive bolt fractures at the site where a groove is designated on the outer cylinder of the explosive bolt with the help of an internal power source generated by an explosive charge. The launch process of satellites lasts longer than the duration of the departure process so that failure due to the strength degradation of the explosive bolt may occur. In this section, we will concentrate on a dynamic reliability analysis of explosive bolts in the launch process of satellites.

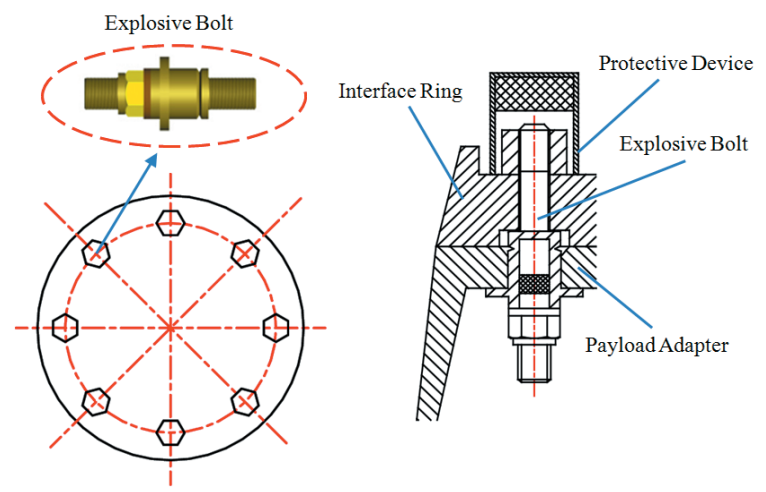

Fig. 3. Structure of the explosive bolt

Due to the randomness of the environmental load in the launch process of satellites and the variation in the manufacturing process of explosive bolts, the stress and the initial strength should be modelled as random variables. In the launch process of satellites, the explosive bolts are mainly used to withstand the random environmental axial forces while the shear forces are sustained by other mechanical components [11]. The distribution of stress can be obtained through a finite element analysis (FEA) of explosive bolts as shown in Fig. 4. The distribution of initial strength can be obtained by experimental tests [11]. The method of constructing the finite element model of bolted joints and the statistical method for the distributions of random variables is found in reference [16]. In addition, Crocombe developed a method for estimating the energy dissipated in the bolted joints of a satellite structure[17]. Nethercot investigated the behaviour of stainless steel bolted connections using the finite element models [18]. Oskouei analysed the stress of aircraft structural double-lapbolted joints by using the finite-element method [19]. Nassar proposed a method of calculating the bearing friction forces on the rotating contact surface of threaded fasteners [20]. In this section, we focus on validating the proposed dynamic reliability models and analysing the influences of the variation in statistical characteristics of material parameters on the reliability and failure rate of explosive bolts.

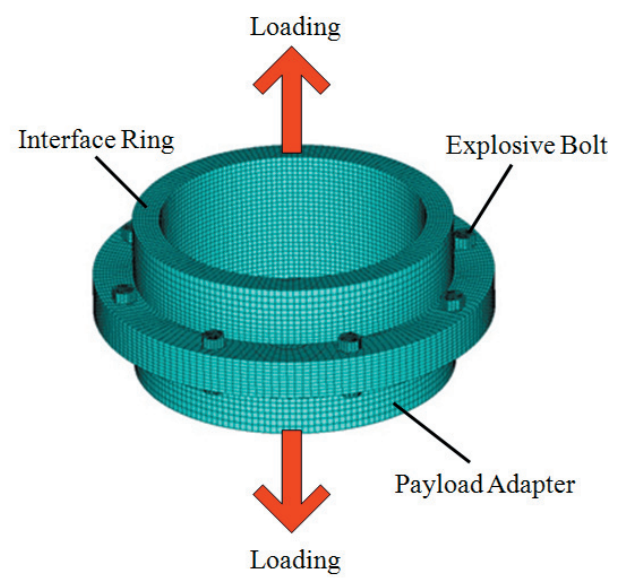

Fig. 4. Finite element model of an explosive bolt

The material parameters of the explosive bolts are given by $m=2, \alpha=1$ and $C=10^{9} \mathrm{MPa}^{2}$. The initial strength of the explosive bolt is normally distributed with a mean value of $\mu\left(r_{0}\right)$ and a standard deviation of $\sigma\left(r_{0}\right)$. The stress at each load application follows the normal distribution with a mean value of $\mu(s)$ and a standard deviation of $\sigma(s)$. The value of the mean value and standard deviation of the initial strength and stress are listed in Table 2.

Table 2. Statistical parameters of stress and initial strength

\begin{tabular}{cccc}
\hline$\mu\left(r_{0}\right)[\mathrm{MPa}]$ & $\sigma\left(r_{0}\right)[\mathrm{MPa}]$ & $\mu(\mathrm{s})[\mathrm{MPa}]$ & $\sigma(s)[\mathrm{MPa}]$ \\
\hline 600 & 20 & 500 & 20 \\
\hline
\end{tabular}

In order to validate the reliability model proposed in Section 1.1, a Monte Carlo simulation for evaluating 
the dynamic reliability of explosive bolts is carried out in this section and the flowchart of the Monte Carlo simulation is shown in Fig. 5. In the Monte Carlo simulation, the stress at each load application is generated according to its distribution and the strength degradation of an explosive bolt sample is simulated based on the degradation mechanism and all the stress generated in its strength degradation path. Therefore, the Monte Carlo simulation represents the actual strength degradation process of explosive bolts. Additionally, the distribution of strength at each load application can be obtained based on Eq. (10). To illustrate the errors that can be caused by using the distribution of strength at each load application in the calculation of reliability, the reliability calculated by using the proposed model, the reliability calculated according to the strength distribution at each load application, and the reliability from the Monte Carlo simulation are shown in Fig. 6.

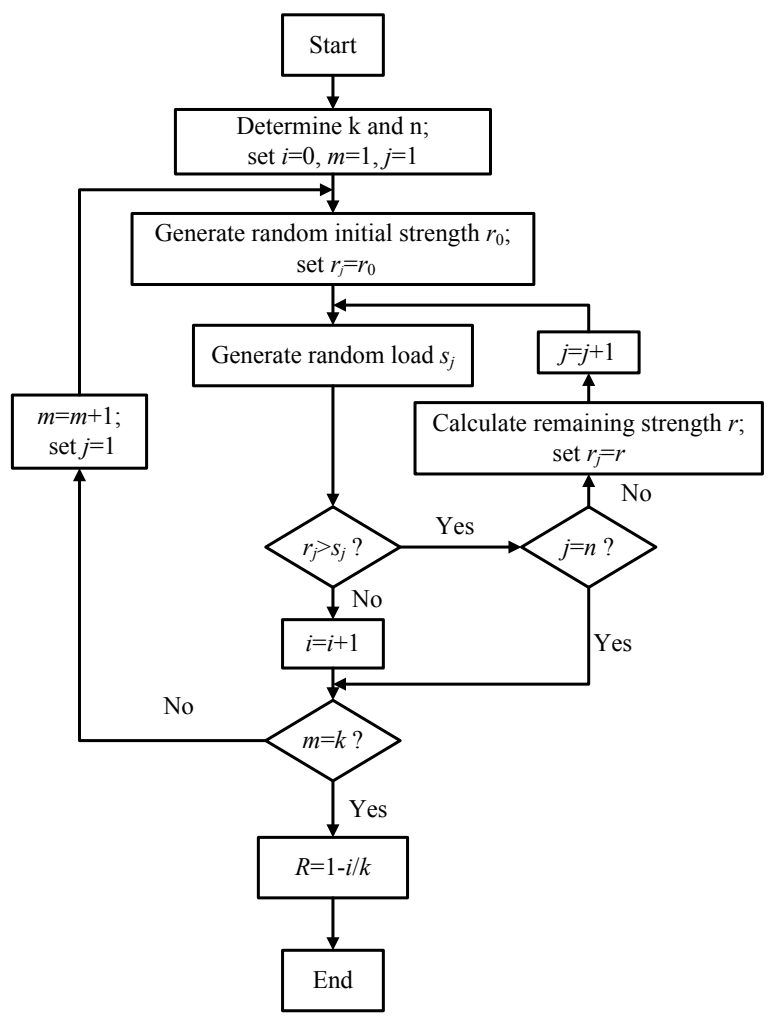

Fig. 5. Flowchart of Monte Carlo simulation

From Fig. 6, we can see that the reliability calculated by using the proposed method in this paper shows good agreement with the results obtained from the Monte Carlo simulation. However, it may result in erroneous calculation of the reliability by using the distribution of strength at each load application, which neglects the correlation between the remaining strength at each load application in a strength degradation path and takes into account non-existent strength degradation paths.

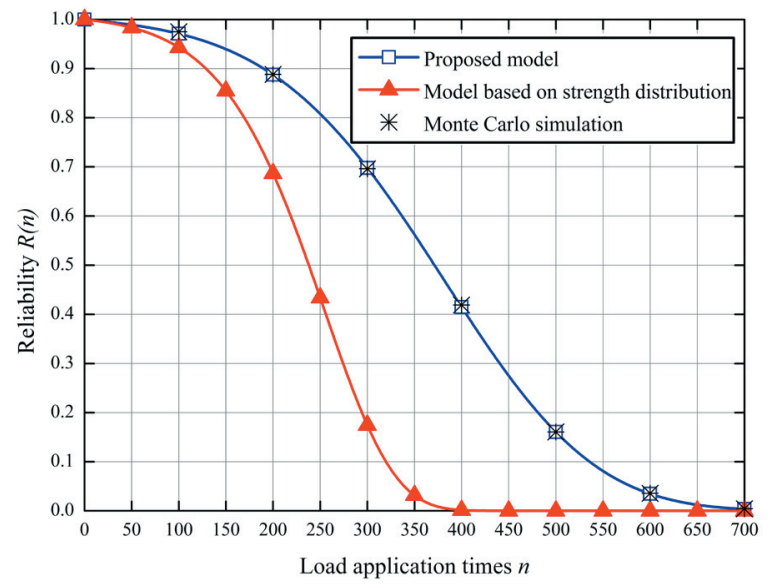

Fig. 6. Comparison between proposed method and Monte Carlo simulation

In addition, to analyse the influences of the variation in statistical parameters of material parameters on the reliability and failure rate of explosive bolts, consider the following four cases:

Case 1: The material parameters of the explosive bolts are given by $m=2, \alpha=1$ and $r_{0}=600 \mathrm{MPa}$. The statistical parameters of stress and $C$ are listed in Table 3. The reliability and failure rate of the explosive bolts with different mean values of $C$ are shown in Figs. 7 and 8 , respectively.

Table 3. Statistical parameters of stress and material parameters C of explosive bolts

\begin{tabular}{ccccc}
\hline & $\mu(\mathrm{s})[\mathrm{MPa}]$ & $\sigma(s)[\mathrm{MPa}]$ & $\mu(\mathrm{C})\left[\mathrm{MPa}^{2}\right]$ & $\sigma(C)\left[\mathrm{MPa}^{2}\right]$ \\
\hline 1 & 500 & 20 & $10^{9}$ & $10^{6}$ \\
\hline 2 & 500 & 20 & $1.5 \times 10^{9}$ & $10^{6}$ \\
\hline 3 & 500 & 20 & $2 \times 10^{9}$ & $10^{6}$ \\
\hline
\end{tabular}

Case 2: The material parameters of the explosive bolts are given by $m=2, \alpha=1$ and $r_{0}=600 \mathrm{MPa}$. The statistical parameters of stress and $C$ are listed in Table 4. The reliability and failure rate of the explosive bolts with different standard deviations of $C$ are shown in Figs. 9 and 10, respectively.

Table 4. Statistical parameters of stress and material parameters C of explosive bolts

\begin{tabular}{ccccc}
\hline & $\mu(\mathrm{s})[\mathrm{MPa}]$ & $\sigma(s)[\mathrm{MPa}]$ & $\mu(\mathrm{C})\left[\mathrm{MPa}^{2}\right]$ & $\sigma(C)\left[\mathrm{MPa}^{2}\right]$ \\
\hline 1 & 500 & 20 & $10^{9}$ & $10^{6}$ \\
\hline 2 & 500 & 20 & $10^{9}$ & $5 \times 10^{6}$ \\
\hline 3 & 500 & 20 & $10^{9}$ & $10^{7}$ \\
\hline
\end{tabular}




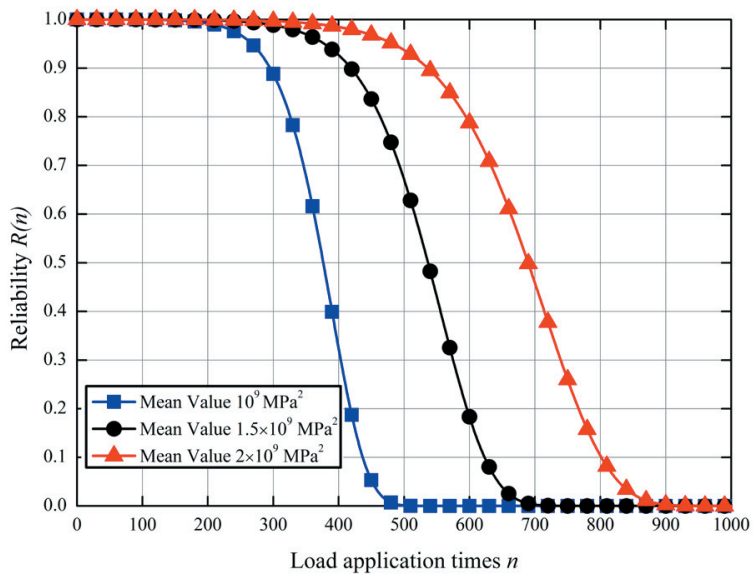

Fig. 7. Reliability of explosive bolts with different mean values of $C$

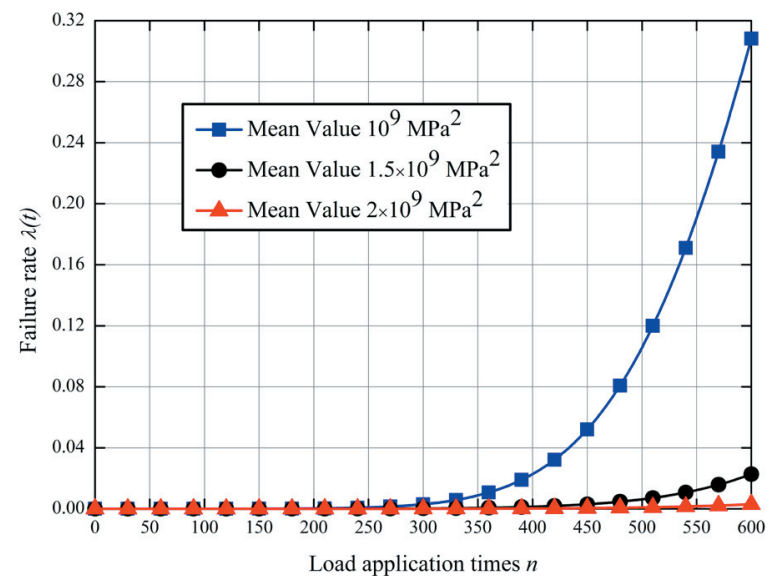

Fig. 8. Failure rate of explosive bolts with different mean values of $\mathrm{C}$

Case 3: The material parameters of the explosive bolts are given by $m=2, \alpha=1$ and $C=10^{9} \mathrm{MPa}^{2}$. The statistical characteristics of both stress and initial strength are listed in Table 5. The reliability and failure rate of the explosive bolts with different mean values of initial strength are shown in Figs. 11 and 12, respectively.

Table 5. Statistical parameters of stress and initial strength of explosive bolts

\begin{tabular}{ccccc}
\hline & $\mu\left(r_{0}\right)[\mathrm{MPa}]$ & $\sigma\left(r_{0}\right)[\mathrm{MPa}]$ & $\mu(\mathrm{s})[\mathrm{MPa}]$ & $\sigma(s)[\mathrm{MPa}]$ \\
\hline 1 & 550 & 30 & 500 & 20 \\
\hline 2 & 600 & 30 & 500 & 20 \\
\hline 3 & 650 & 30 & 500 & 20 \\
\hline
\end{tabular}

Case 4: The material parameters of the explosive bolts are given by $m=2, \alpha=1$ and $C=10^{9} \mathrm{MPa}^{2}$. The statistical characteristics of both stress and initial strength are listed in Table 6. The reliability and failure

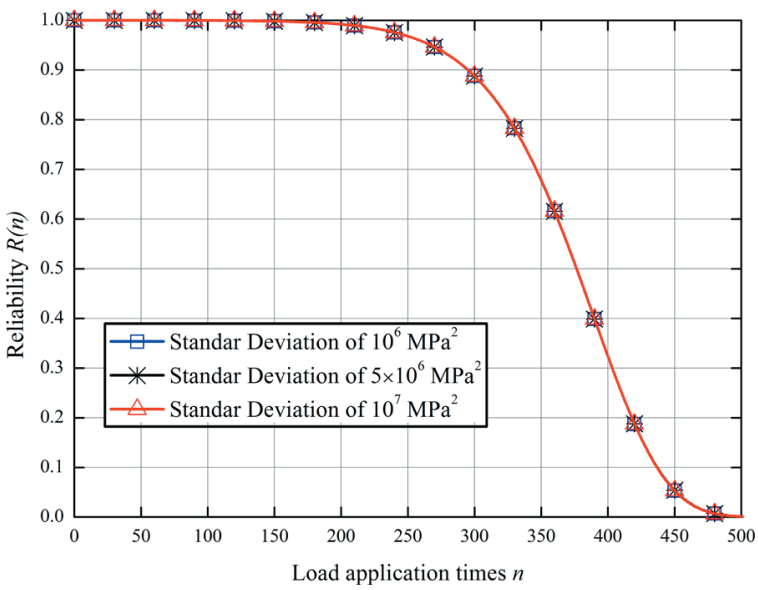

Fig. 9. Reliability of explosive bolts with different dispersions of $C$

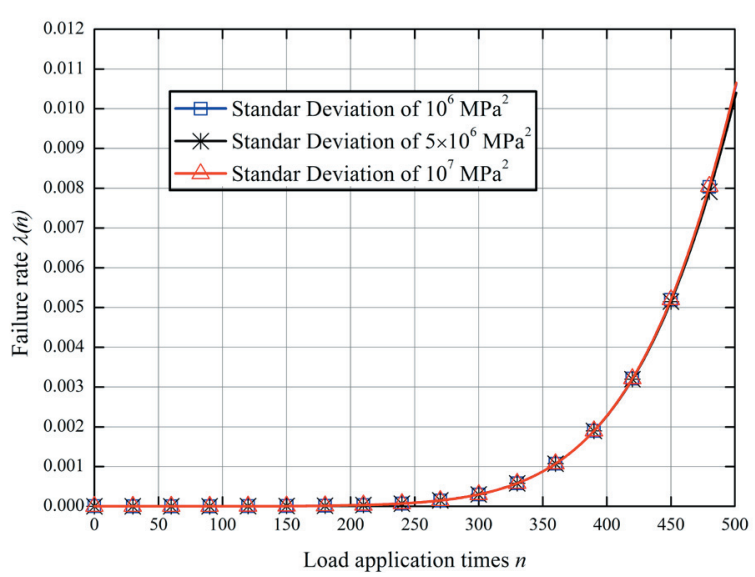

Fig. 10. Failure rate of explosive bolts with different dispersions of $\mathrm{C}$

rate of the explosive bolts with different standard deviation of initial strength are shown in Figs. 13 and 14 , respectively.

Table 6. Statistical parameters of stress and initial strength of explosive bolts

\begin{tabular}{ccccc}
\hline & $\mu\left(r_{0}\right)[\mathrm{MPa}]$ & $\sigma\left(r_{0}\right)[\mathrm{MPa}]$ & $\mu(\mathrm{s})[\mathrm{MPa}]$ & $\sigma(s)[\mathrm{MPa}]$ \\
\hline 1 & 600 & 20 & 500 & 20 \\
\hline 2 & 600 & 30 & 500 & 20 \\
\hline 3 & 600 & 40 & 500 & 20 \\
\hline
\end{tabular}

Case 5: The material parameters of the explosive bolts are given by $m=2, \alpha=1$ and $C=109 \mathrm{MPa}^{2}$. The statistical parameters of stress and $\mathrm{r} 0$ are listed in Table 7. The reliability of the explosive bolts with different dispersions of stress are shown in Fig. 15. 


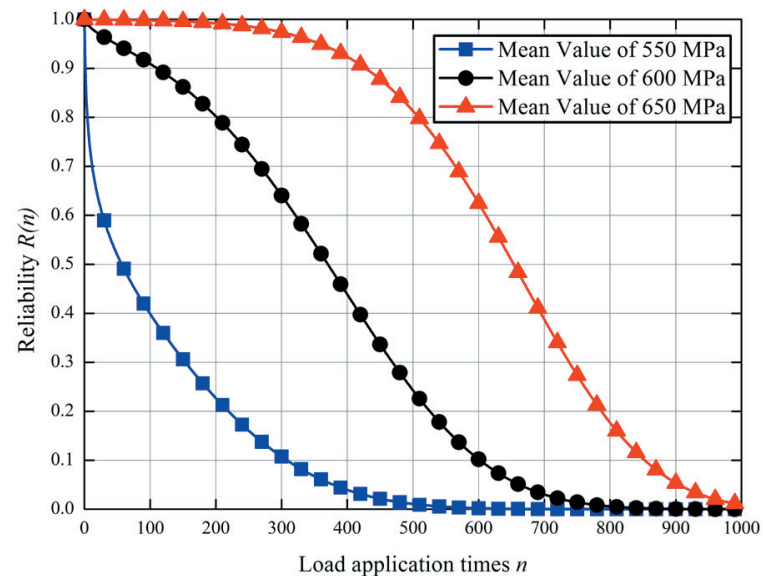

Fig. 11. Reliability of explosive bolts with different mean values of initial strength

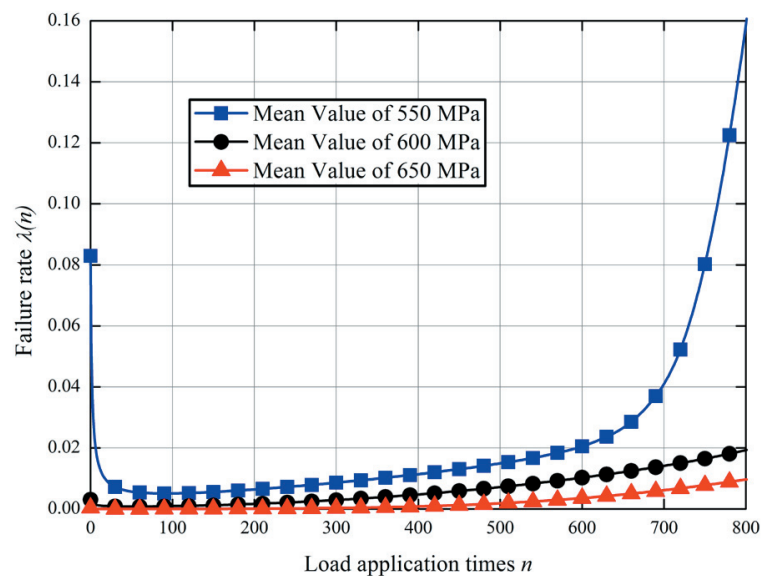

Fig. 12. Failure rate of explosive bolts with different mean values of initial strength

Table 7. Statistical parameters of stress and material parameters C of explosive bolts

\begin{tabular}{ccccc}
\hline & $\mu(s)[\mathrm{MPa}]$ & $\sigma(s)[\mathrm{MPa}]$ & $\mu\left(r_{0}\right)[\mathrm{MPa}]$ & $\sigma\left(r_{0}\right)[\mathrm{MPa}]$ \\
\hline 1 & 500 & 10 & 600 & 30 \\
\hline 2 & 500 & 20 & 600 & 30 \\
\hline 3 & 500 & 30 & 600 & 30 \\
\hline
\end{tabular}

From Figs. 7 to 12, it can be inferred that the mean value of the initial strength and $C$ has a strong influence on the reliability and failure rate of explosive bolts. The reliability increases and the failure rate decreases with the increase in the mean value of the initial strength and $C$. In addition, the dispersion of $C$ has a negligible influence on the reliability and failure rate of explosive bolts and can therefore be neglected in the reliability analysis of explosive bolts. Thus, Eqs. (12) and (13) can be rewritten as follows:

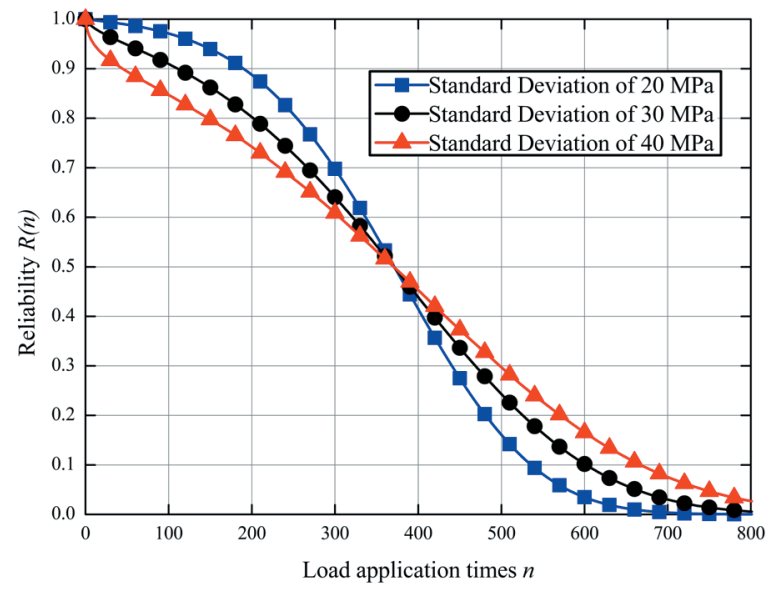

Fig. 13. Reliability of explosive bolts with different dispersions of initial strength

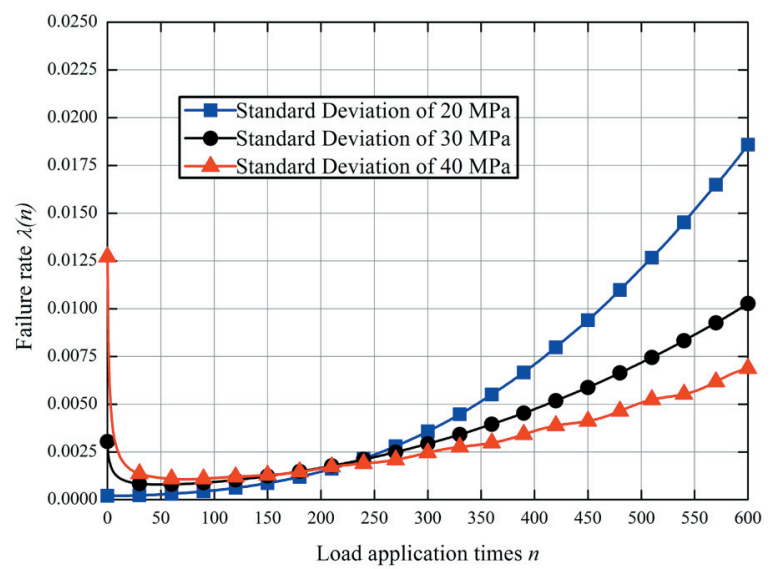

Fig. 14. Failure rate of explosive bolts with different dispersions of initial strength

$$
\begin{aligned}
& R(n)=\int_{-\infty}^{\infty} f_{r_{0}}\left(r_{0}\right)\left\{\prod_{i=0}^{n-1}\left[\int_{-\infty}^{r_{0}\left(1-\frac{i \int_{-\infty}^{\infty} s^{m} f_{s}(s) \mathrm{d} s}{C}\right)^{a}} f_{s}(s) \mathrm{d} s\right]\right\} d r_{0}, \\
& h(n)=\left\{\int_{-\infty}^{\infty} f_{r_{0}}\left(r_{0}\right)\left\{\prod_{i=0}^{n-1}\left[\int_{-\infty}^{r_{0}\left(1-\frac{i \int_{-\infty}^{\infty} s^{m} f_{s}(s) \mathrm{d} s}{C}\right)^{a}} f_{s}(s) \mathrm{d} s\right]\right\} d r_{0}-\right. \\
& \left.-\int_{-\infty}^{\infty} f_{r_{0}}\left(r_{0}\right)\left\{\prod_{i=0}^{n}\left[\int_{-\infty}^{r_{0}\left(1-\frac{i \int_{-\infty}^{\infty} s^{m} f_{s}(s) \mathrm{d} s}{C}\right)^{a}} f_{s}(s) \mathrm{d} s\right]\right\} d r\right\} / \\
& /\left\{\int_{-\infty}^{\infty} f_{r_{0}}\left(r_{0}\right)\left\{\prod_{i=0}^{n-1}\left[\int_{-\infty}^{r_{0}\left(1-\frac{i \int_{-\infty}^{\infty} s^{m} f_{s}(s) \mathrm{d} s}{C}\right)^{a}} f_{s}(s) \mathrm{d} s\right]\right\} d r_{0}\right\} .
\end{aligned}
$$

In addition, it is traditionally considered that a large dispersion leads to lower reliability. However, 
from Figs. 13 and 14, it can be seen that the dispersion of initial strength has different influences on the reliability and failure rate of explosive bolts at different stages of their lifetime. This is because during the initial period of lifetime, the reliability is high and a large dispersion of initial strength increases the possibility that the remaining strength will have a small value, which leads to low reliability. At the stage of lifetime when the reliability is low, a large dispersion of initial strength increases the possibility that the remaining strength takes a large value, which leads to a high reliability.

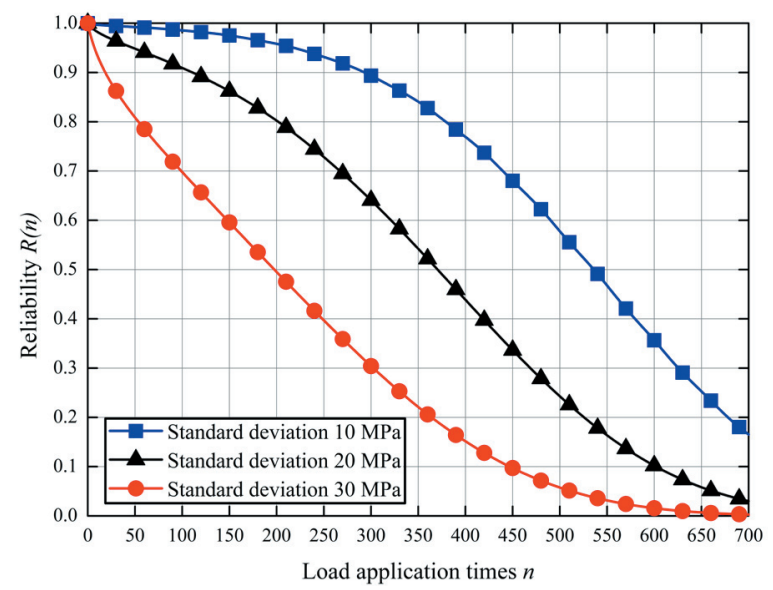

Fig. 15. Reliability of explosive bolts under stress with different standard deviations

From Fig. 15, it can be inferred that the dispersion of stress has a strong influence on the dynamic reliability of explosive bolts. The reliability decreases with the increase in the dispersion of stress. This is because a large dispersion of stress increases the possibility that the stress will exceed the remaining strength in the load application process.

\section{DYNAMIC RELIABILITY ANALYSIS OF MECHANICAL COMPONENTS WITH RESPECT TO TIME}

In this section, dynamic reliability models with respect to time are established based on the models proposed in Section 1.1, which take account of the failure mechanism and the stochastic strength degradation path. Moreover, numerical examples are given to analyse the influences of the statistical characteristics of initial strength on the dynamic behaviour of reliability and on the failure rate of mechanical components.

\subsection{Dynamic Reliability Models with Respect to Time}

As mentioned above, for mechanical components with the failure mode of fatigue, the assumption of continuous statistical characteristics of load with respect to time indicates infinite times of load application in an infinitesimal time interval $\Delta t$, which is unreasonable in reality. The load process should be characterised by the load application times and the magnitude of load. Therefore, the reliability models proposed in Section 1.1 provide a basis for developing reliability models with respect to time. When the relationship between load application times and time is obtained, dynamic reliability of components with respect to time can be further developed based on the models proposed in Section 1.1. Provided that the load application times in a time interval is deterministic, which is expressed by using a deterministic function $n=f_{t}(t)$ with respect to time, the dynamic reliability of mechanical components can be calculated as follows:

$\left.R(t)=\int_{-\infty}^{\infty} f_{r_{0}}\left(r_{0}\right)\left\{\prod_{i=0}^{f_{f}(t)-1}\left[\int_{-\infty}^{r_{0}\left(1-\frac{i-\infty}{C} \int_{-\infty}^{\infty} s^{m} f_{s}(s) \mathrm{d} s\right.}\right)^{a} f_{s}(s) \mathrm{d} s\right]\right\} d r_{0}$.

However, in some situations, the occurrence times of load are random, which can only be analysed by using the stochastic process theory. In practice, it has been proved that the Poisson process is an effective stochastic process to describe the random occurrence times of random load in a time interval. According to the Poisson process theory, the probability that the random load appears $n$ times in a time interval $t$ can be given by reference [6]:

$\operatorname{Pr}[n(t)-n(0)=n]=\frac{\left(\int_{0}^{t} \lambda(t) d t\right)^{n}}{n !} \exp \left(-\int_{0}^{t} \lambda(t) \mathrm{d} t\right)$,

where $\lambda(t)$ is the intensity of the Poisson process. For a mechanical component with deterministic initial strength $r$, according to the total probability theorem, the reliability in the time interval of $t$ can be expressed as follows:

$$
\begin{aligned}
R(t) & =\sum_{k=0}^{\infty} P(n(t)=k) R(k)= \\
& =\exp \left(-\int_{0}^{t} \lambda(t) \mathrm{d} t\right)+\sum_{n=1}^{\infty} \frac{\left(\int_{0}^{t} \lambda(t) \mathrm{d} t\right)^{n}}{n !} \times \\
& \times \exp \left(-\int_{0}^{t} \lambda(t) \mathrm{d} t\right)\left\{\prod_{i=0}^{n-1}\left[\int_{-\infty}^{r\left(1-\frac{i \int_{-\infty}^{\infty} s^{m} f_{s}(s) \mathrm{d} s}{C}\right)^{a}} f_{s}(s) \mathrm{d} s\right]\right\} .
\end{aligned}
$$


When considering the distribution of initial strength characterised by its pdf of $f_{r}(r)$, the reliability can be obtained by using the Bayes law for continuous variables as follows:

$$
\begin{aligned}
& R(t)=\int_{-\infty}^{\infty} f_{r}(r) \exp \left(-\int_{0}^{t} \lambda(t) \mathrm{d} t\right)+\sum_{n=1}^{\infty} \frac{\left(\int_{0}^{t} \lambda(t) \mathrm{d} t\right)^{n}}{n !} \times \\
& \times \exp \left(-\int_{0}^{t} \lambda(t) \mathrm{d} t\right)\left\{\prod_{i=0}^{n-1}\left[\int_{-\infty}^{r\left(1-\frac{i \int_{-\infty}^{\infty} s^{m} f_{s}^{(s) \mathrm{d} s}}{C}\right)^{a}} f_{s}(s) \mathrm{d} s\right]\right\} d r .
\end{aligned}
$$

Correspondingly, the failure rate of the component can be written as:

$$
\begin{aligned}
h(t) & =\left\{\lambda ( t ) \int _ { - \infty } ^ { \infty } f _ { r } ( r ) \left\{1-\sum_{n=1}^{\infty} \frac{\left(\int_{0}^{t} \lambda(t) \mathrm{d} t\right)^{n-1}}{n !}\left[n-\int_{0}^{t} \lambda(t) \mathrm{d} t\right] \times\right.\right. \\
& \left.\left.\times\left\{\prod_{i=0}^{n-1}\left[\int_{-\infty}^{r\left(1-\frac{i \int_{-\infty}^{\infty} s^{m} f_{s}(s) \mathrm{d} s}{c}\right)^{a}} f_{s}(s) \mathrm{d} s\right]\right\}\right\} d r\right\} / \\
& /\left\{\int _ { - \infty } ^ { \infty } f _ { r } ( r ) \left\{1+\sum_{n=1}^{\infty} \frac{\left(\int_{0}^{t} \lambda(t) \mathrm{d} t\right)^{n}}{n !} \times\right.\right. \\
& \left.\left.\times\left\{\prod_{i=0}^{n-1}\left[\int_{-\infty}^{r\left(1-\frac{i]_{-\infty}^{\infty} s^{m} f_{s}(s) \mathrm{d} s}{C}\right)^{a}} f_{s}(s) \mathrm{d} s\right]\right\}\right\} d r\right\} .
\end{aligned}
$$

Although the dynamic reliability model with respect to time is developed based on the theory of the Poisson process, it should be noted that the reliability model proposed in section 2.1 can be easily extended to other dynamic reliability models with respect to time, as long as the statistical characteristics of the load application times are obtained. In addition, from the derivation of Eqs. (17) and (18), it can be seen that the proposed dynamic reliability model with respect to time takes the stochastic strength degradation path of mechanical components into consideration. To illustrate the error caused by estimating reliability based on the strength distribution at each load application, numerical examples will be given in the following section.

\subsection{Numerical Examples}

Consider the explosive bolts operating under the application of random load, whose occurrence times follow the Poisson process with an intensity of $0.6 \mathrm{~h}^{-1}$. The initial strength and the stress follow the normal distribution. The material parameters of the explosive bolts are given by $m=2, \alpha=1$ and $C=10^{8} \mathrm{MPa}^{2}$. The statistical characteristics of stress and initial strength are listed in Table 8 . The reliability calculated by using the models proposed in this paper and the reliability calculated based on the distribution of strength at each load application are shown in Fig. 16.

Table 8. Statistical parameters of stress and initial strength of explosive bolts

\begin{tabular}{cccc}
\hline$\mu\left(r_{0}\right)[\mathrm{MPa}]$ & $\sigma\left(r_{0}\right)[\mathrm{MPa}]$ & $\mu(\mathrm{s})[\mathrm{MPa}]$ & $\sigma(s)[\mathrm{MPa}]$ \\
\hline 400 & 30 & 300 & 20 \\
\hline
\end{tabular}

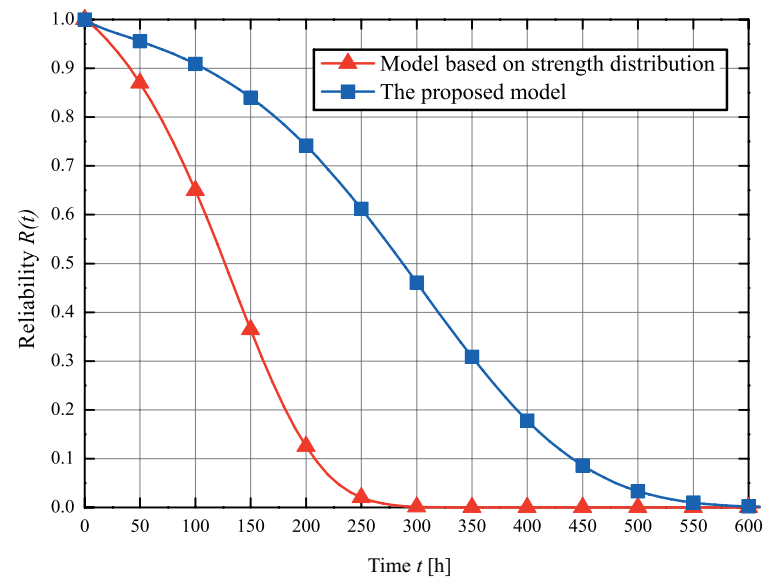

Fig. 16. Comparison between the reliability calculated by using the proposed method and the reliability calculated based on the strength distribution at each load application

From Fig. 16, we can see that the proposed reliability models can be used to represent the variation in reliability with time. In addition, it leads to lower reliability to use the distribution of strength at each load application. The error is caused by taking into account strength degradation paths which do not exist in practice. Therefore, dynamic reliability models with respect to time should be developed based on the strength degradation path rather than on the distribution of strength at each load application.

In order to analyse the influences of the mean value and dispersion of initial strength on the reliability and failure rate of explosive bolts, consider the following two cases:

Case 1: The material parameters of the explosive bolts are given by $m=2, \alpha=1$ and $C=10^{8} \mathrm{MPa}^{2}$. The statistical characteristics of both stress and initial strength are listed in Table 9. The reliability and failure rate of the explosive bolts with different mean values of initial strength are shown in Figs. 17 and 18, respectively.

Case 2: The material parameters of the explosive bolts are given by $m=2, \alpha=1$ and $C=10^{8} \mathrm{MPa}^{2}$. 


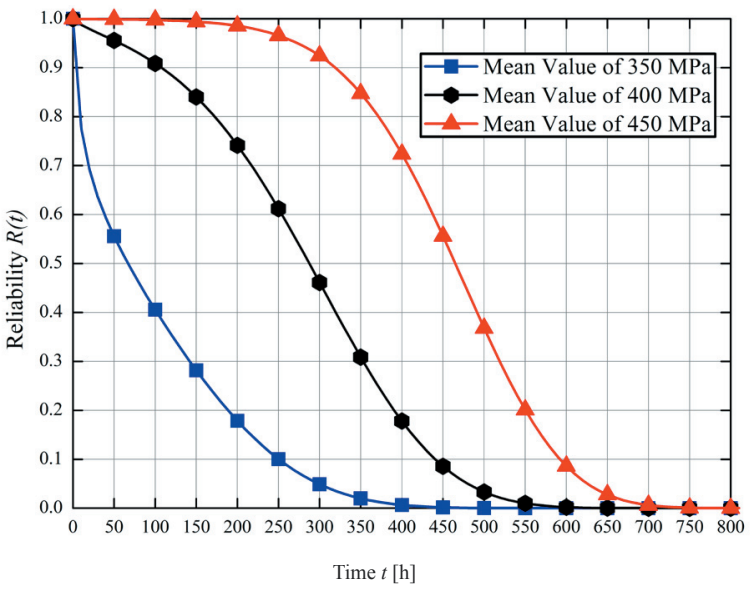

Fig. 17. Reliability of explosive bolts with different mean values of initial strength

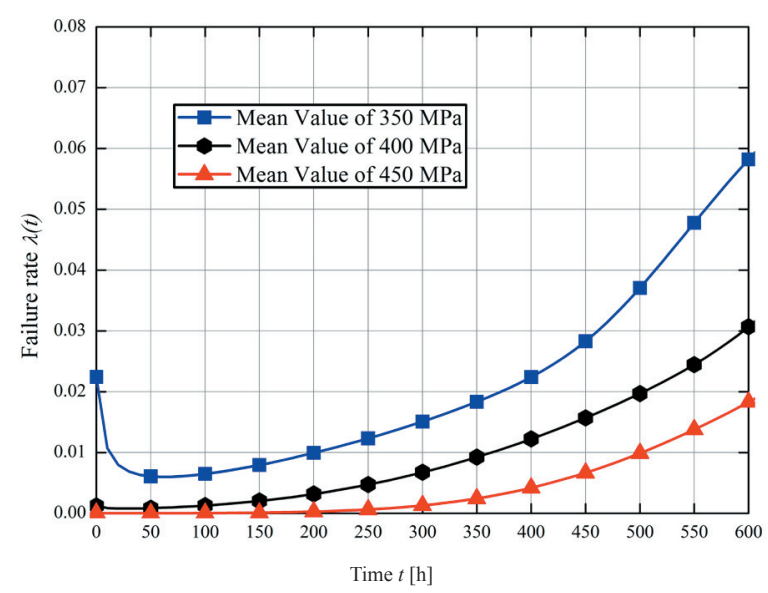

Fig. 18. Failure rate of explosive bolts with different mean values of initial strength

The statistical characteristics of both stress and initial strength are listed in Table 10. The reliability and failure rate of the explosive bolts with different standard deviations of initial strength are shown in Figs. 19 and 20, respectively.

Table 9. Statistical parameters of stress and inistial strength of explosive bolts

\begin{tabular}{ccccc}
\hline & $\mu\left(r_{0}\right)[\mathrm{MPa}]$ & $\sigma\left(r_{0}\right)[\mathrm{MPa}]$ & $\mu(\mathrm{s})[\mathrm{MPa}]$ & $\sigma(s)[\mathrm{MPa}]$ \\
\hline 1 & 350 & 30 & 300 & 20 \\
\hline 2 & 400 & 30 & 300 & 20 \\
\hline 3 & 450 & 30 & 300 & 20 \\
\hline
\end{tabular}

From Figs. 17 to 20, we can see that the proposed dynamic reliability models can be used to analyse the dynamic characteristics of reliability and failure rate and quantitatively analyse the influence

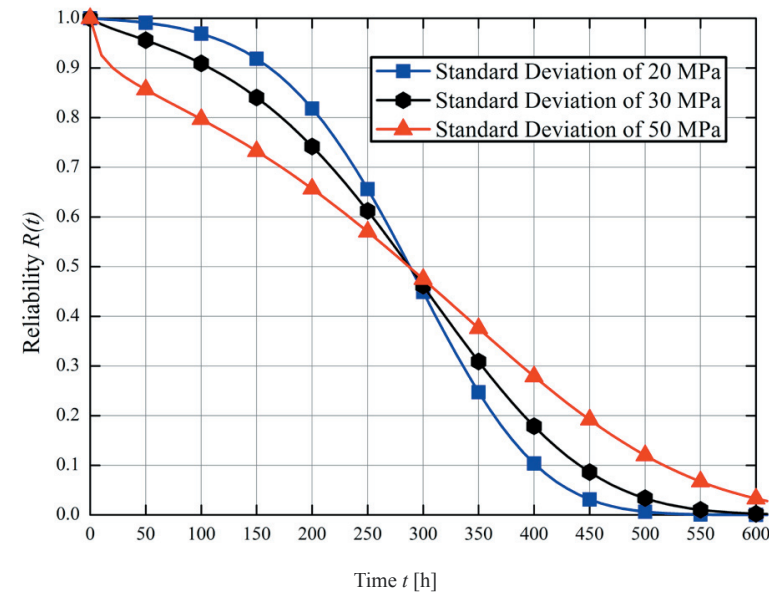

Fig. 19. Reliability of explosive bolts with different dispersions of initial strength

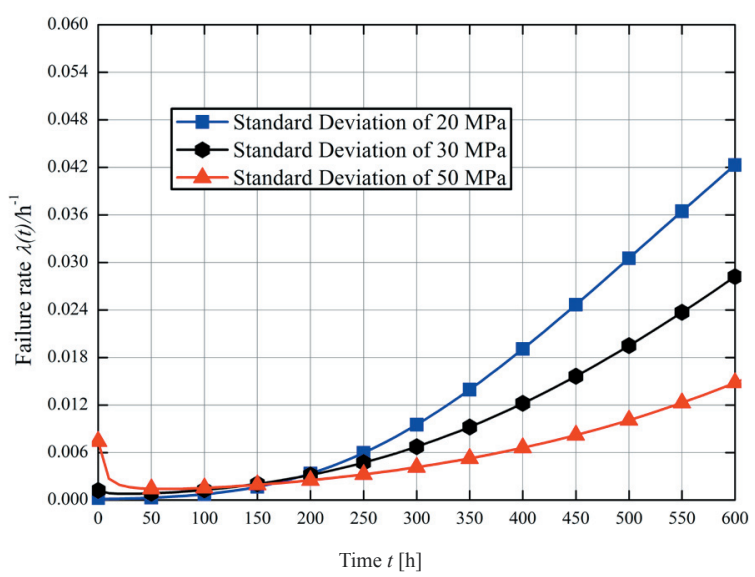

Fig. 20. Failure rate of explosive bolts with different dispersions of intial strength

of the variation in statistical parameters of material parameters on the reliability and failure rate. The reliability increases and failure rate decreases with the increase in the mean value of initial strength. Moreover, the dispersion of initial strength has different influences on the reliability and failure rate of the explosive bolts at different periods of lifetime. In addition, the bathtub curve is always used to represent the variation in the failure rate of mechanical components with time as shown in Fig. 21.

Table 10. Statistical parameters of stress and initial strength of explosive bolts

\begin{tabular}{ccccc}
\hline & $\mu\left(r_{0}\right)[\mathrm{MPa}]$ & $\sigma\left(r_{0}\right)[\mathrm{MPa}]$ & $\mu(\mathrm{s})[\mathrm{MPa}]$ & $\sigma(s)[\mathrm{MPa}]$ \\
\hline 1 & 400 & 20 & 300 & 20 \\
\hline 2 & 400 & 30 & 300 & 20 \\
\hline 3 & 400 & 50 & 300 & 20 \\
\hline
\end{tabular}




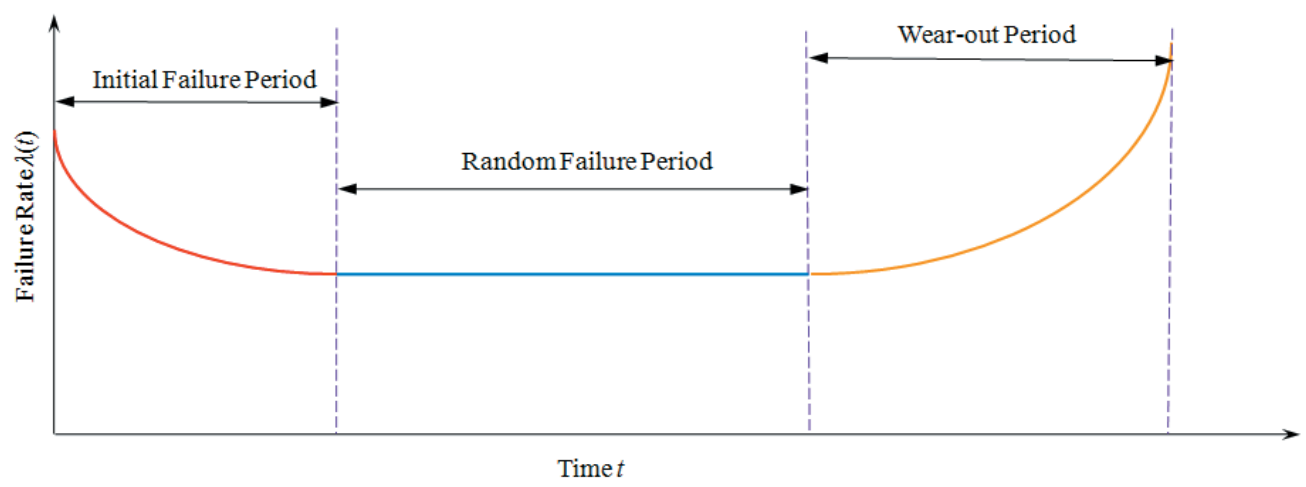

Fig. 21. Schematic bathtub curve of mechanical components

From Figs. 18 and 20, it can be seen that the failure rate curve obtained from the reliability model developed in this paper is consistent with the bathtub curve theory. Furthermore, the slope of the failure rate curve for mechanical components in the random failure period tends to decrease with the increase in the mean value and the dispersion of initial strength.

\section{CONCLUSIONS AND FUTURE WORK}

Dynamic reliability models based on strength degradation paths are developed in this paper. Owing to the difficulty in mathematically describing the strength degradation path, which is caused by the randomness of loads applied to mechanical components, the distribution of strength at each load application is always used to analyse the dynamic reliability of the mechanical components. However, it may lead to large errors in the reliability calculation to treat the uncertainty of strength in this way due to neglecting the correlation with the remaining strength at each load application in a strength degradation path.

The proposed reliability models can be used to quantitatively analyse the influence of the variation in statistical parameters of material parameters on the dynamic characteristics of reliability and on the failure rate of mechanical components. Traditionally, it is considered that a large dispersion of initial strength results in a lower reliability. However, when considering strength degradation, the dispersion of initial strength has different influences on the reliability of mechanical components at different stages of lifetime. Moreover, it should be noted that the statistical characteristics of initial strength have strong influences on the dynamic behaviour of the failure rate of mechanical components. The slope of the failure rate curve for mechanical components in the random failure period tends to decrease with the increase in the mean value and the dispersion of initial strength.

Further work is in progress to include other variables in the reliability models to achieve more accurate predictive results. Moreover, extensions of the proposed method to problems of reliability-based design optimisation are currently being investigated by the authors.

\section{ACKNOWLEDGEMENTS}

This work was supported by the National Science Foundation of China under Contract No. 11072123, the National High Technology Research and Development Program of China (863 Program) under Contract No. 2009AA04Z401, the Major State Basic Research Development Program of China (973 Program), and the Project sponsored by SRF for ROCS, SEM.

\section{REFERENCES}

[1] Dasic, P., Natsis, A., Petropoulos, G. (2008). Models of reliability for cutting tools: Examples in manufacturing and agricultural engineering. Strojniški vestnik Journal of Mechanical Engineering, vol. 54, no. 2, p. 122-130.

[2] Li, Y.M. (2008). Stiffness analysis for a 3-PUU parallel kinematic machine. Mechanism and Machine Theory, vol. 43, no. 2, p. 186-200, DOI:10.1016/j. mechmachtheory.2007.02.002.

[3] Li, C.Q. (1994). Probability of plastic collapse of a structural system under nonstationary load processes. Computers and Structures, vol. 52, no. 1, p. 69-78, DOI:10.1016/0045-7949(94)90257-7.

[4] Martin, P. (1998). A review of mechanical reliability. Journal of Process Mechancial Engineering, vol. 212, no. E4, p. 281-287, DOI:10.1243/0954408981529484.

[5] Levis, E.E. (2001). A Load-capacity interference model for common-mode failures in 1-out-of-2: G systems. 
IEEE Transactions on Reliability, vol. 50, no. 1, p. $47-$ 51, DOI:10.1109/24.935017.

[6] Geidl, V., Saunders, S. (1987). Calculation of reliability for time-varying loads and resistances. Structural Safety, vol. 4, no. 4, p. 285-292, DOI:10.1016/01674730(87)90003-8.

[7] Somasundaram, S., Ausdin Mohana Dhas, D. (1997). Reliability of a dynamic n-unit shared load parallel system under different failure times. Microelectronics and Reliability, vol. 37, no. 5, p. 869-871, DOI:10.1016/S0026-2714(96)00100-X.

[8] Van Noortwijk, J.M., Van der Weide, J.A.M., Kallen, M.J., Pandey, M.D. (2007). Gamma processes and peaks-over-threshold distributions for time-dependent reliability. Reliability Engineering and System Safety, vol. 92, no. 12, p. 1651-1658, DOI:10.1016/j. ress.2006.11.003.

[9] Labeaua, P. E., Smidts, C., Swaminathan, S. (2000). Dynamic reliability: towards an integrated platform for probabilistic risk assessment. Reliability Engineering and System Safety, vol. 68, no. 3, p. 219-254, DOI:10.1016/S0951-8320(00)00017-X.

[10] Zhang, Y., Tong, J., Zhou, Y., Cai, Q. (2012). Review of dynamic reliability methodology in probabilistic safety assessments of nuclear power plants. Atomic Energy Science and Technology, vol. 46, no. 4, p. 472-479. (in Chinese)

[11] Slak, A., Tavčar, J., Duhovnik, J. (2011). Application of Genetic Algorithm into Multicriteria Batch Manufacturing Scheduling, Strojniški vestnik - Journal of Mechanical Engineering, vol. 57, no. 2, p. 110-124, DOI:10.5545/sv-jme.2010.122.

[12] Barkallah, M., Louati, J., Haddar, M. (2012). Evaluation of Manufacturing Tolerance Using a Statistical Method and Experimentation. International Journal of Simulation Modelling, vol. 11, no. 1, p. 5-16, DOI:10.2507/IJSIMM11(1)1.194.
[13] Gu, Y.A., An, W.G., An, H. (2007). Structural reliability analysis under dead load and fatigue load. Acta Armamentar, vol. 28, no. 12, p. 1473-1477.

[14] Dattoma, V., Giancane, S., Nobile, R., Panella, F.W. (2006). Fatigue life prediction under variable loading based on a new non-linear continuum damage mechanics model. International Journal of Fatigue, vol. 28, no. 2, p. 89-95, DOI:10.1016/j. ijfatigue.2005.05.001.

[15] Yuan, J.J. (2004). Design and Analysis of Satellite Structures. China Astronautic Publishing House, Beijing.

[16] Kim, J., Yoon, J.C., Kang B.S. (2007). Finite element analysis and modeling of structure with bolted joints. Applied Mathematical Modelling, vol. 31, no. 5, p. 895-911, DOI:10.1016/j.apm.2006.03.020.

[17] Crocombe, A.D., Wang, R., Richardson, G., Underwood,C.I. (2006). Estimating the energy dissipated in a bolted spacecraft at resonance. Computers and Structures, vol. 84, no. 5-6, p. 340-350, DOI:10.1016/j.compstruc.2005.09.024.

[18] Nethercot, D.A., Salih, E.L., Gardner, L. (2011). Behaviour and design of stainless steel bolted connections. Advances in Structural Engineering, vol. 14, no. 4, p. 647-658, DOI:10.1260/13694332.14.4.647.

[19] Oskouei, R.H., Keikhosravy, M., Soutis, C. (2009). Estimating clamping pressure distribution and stiffness in aircraft bolted joints by finite-element analysis. Proceedings of the Institution of Mechanical Engineers, Part G: Journal of Aerospace Engineering, vol. 223, no. 7, p. 863-871, DOI:10.1243/09544100JAERO596.

[20] Nassar, S.A., Barber, G. C., Barber, G. C., Zuo, D. (2005). Bearing Friction Torque in Bolted Joints. Tribology Transactions, vol. 48, no. 1, p. 69-75, DOI:10.1080/05698190590899967. 